\title{
Instanton approach to the Langevin motion of a particle in a random potential
}

\author{
A. V. Lopatin ${ }^{1}$ and V. M. Vinokur ${ }^{2}$ \\ ${ }^{1}$ Department of Physics, Rutgers University, Piscataway, New Jersey 08854 \\ ${ }^{2}$ Material Science Division, Argonne National Laboratory, Argonne, Illinois 60439
}

(November 21, 2018)

\begin{abstract}
We develop an instanton approach to the non-equilibrium dynamics in one-dimensional random environments. The long time behavior is controlled by rare fluctuations of the disorder potential and, accordingly, by the tail of the distribution function for the time a particle needs to propagate along the system (the delay time). The proposed method allows us to find the tail of the delay time distribution function and delay time moments, providing thus an exact description of the long-time dynamics. We analyze arbitrary environments covering different types of glassy dynamics: dynamics in a short-range random field, creep, and Sinai's motion.
\end{abstract}

Introduction-One dimensional driven dynamics in a random environment attracts a great deal of current attention. The motivation of the interest is two-fold: First, the propagation of a particle through a 1D random field has become a paradigm for a general out-of-equilibrium stochastic processes in random systems capturing all effects of glassy dynamics including aging and memory effectst 1 . Second, a particle moving in a 1D random potential models straightforwardly a variety of physical systems ranging from dislocations and charge density waves in solids, spin-chain dynamics and domain growth, to protein molecules and bacterial coloniest 4 6. The attraction of the 1D models is thus that while allowing for an analytical treatment (and often even for a full analytical solution) they also offer an insight into generic basic properties of the wealth of glassy out-of -equilibrium systems. Indeed, even the simplest $1 \mathrm{D}$ models with the Gaussian correlated potential $v(x)$, with $\langle v(x)\rangle=0$, $\left\langle(v(x)-v(0))^{2}\right\rangle=\kappa|x|^{\gamma}$ exhihit a striking generality and diversity of glassy behaviors

The approach employed in 8.9 enables the exact derivation of the particle velocity, but it does not allow for a complete dynamic description of the system - for example, in terms of velocity cumulants and/or correlation functions. Taking a kinetic view on the problem one can characterize the dynamic properties of a random system by the probability distribution for the particle velocity or, equivalently, by, the probability distribution for the particle delay time $\tau$. The latter is defined as an average time that a driven particle spends to propagate through the sample, and the corresponding distribution function $P(\tau)$ characterizes completely the transport properties of the $1 \mathrm{D}$ system involved.

The exact expressions for a particle velocity derived in 8 . 9 indicate that the system dynamics is governed by rare fluctuations of the random potential, i.e. by the tail of the delay time distribution function. Thus the instanton solution for the Langevin equation in the presence of an external force offers a most adequate description of the long time glassy dynamics in an arbitrary 1D random environment. In this Ietter we apply the instanton method developed earlier in to find the asymptotic behavior of the delay time distribution function $P(\tau)$ at large $\tau$. In general, the idea of the instanton method is to pick up the largest contribution to the functional integral coming from an optimal configuration instead of implementing the complete integration. In our case of low enough temperatures, the delay time is mainly determined by the transition over the largest barrier in the system. Since this time is exponentially large, one expects that the delay time averaged over the random potential (or any of its higher moments) is determined by the saddle point trajectory of some effective action controlling this exponential behavior, and, thus, it can be most appropriately found by the instanton method indeed. Further, knowing all the moments of the delay time one can reconstruct the large $\tau$ asymptotics of the distribution function $P(\tau)$.

The distribution function $P(\tau)$ depends strongly on the specific form of the correlation function of the random potential $u(x)$. We restrict ourselves to the Gaussian disorder with the correlation function

$$
\left\langle v\left(x_{1}\right) v\left(x_{2}\right)\right\rangle_{d}=u\left(x_{1}-x_{2}\right), \quad\langle v(x)\rangle=0 .
$$

We use also the function

$$
K(x)=\left\langle(v(0)-v(x))^{2}\right\rangle_{d}=2(u(0)-u(x)),
$$

which is more convenient in the case of the long-range correlated potential. In the case of the short-range correlated potential, when $u(x)$ monotonically decreases to zero as a function of $|x|$, we find that the delay time distribution is log-normal

$$
\ln \tilde{P}(Y)=-\frac{T^{2} Y^{2}}{4 u(0)},
$$

where $Y=\ln \tau$. The distribution function $\tilde{P}(Y)$ is defined as the distribution function of $\ln \tau$, being therefore related to the distribution function $P(\tau)$ by

$$
P(\tau)=\frac{1}{\tau} \tilde{P}(\ln \tau)
$$

In the case of the long-range correlated potential $K(x)=$ $\kappa x^{\gamma}$, with $\kappa>0$ and $0<\gamma<1$, the distribution function essentially depends on the applied field $E$ : 


$$
\ln \tilde{P}(Y)=-\frac{2}{\kappa}\left[\frac{T Y}{2-\gamma}\right]^{2-\gamma}\left[\frac{E}{\gamma}\right]^{\gamma} .
$$

And, finally, for the so-called Sinai model $K(x)=\kappa x$ we obtain:

$$
P(\tau) \sim \tau^{-2 T E / \kappa-1,}
$$

which agrees with the result found earlier in Ref. alsot)

The model and results- Now let us turn to the detailed calculations. The dynamics of a classical particle in a random potential is described by the Langevin equation

$$
\Gamma^{-1} \partial_{t} x(t)=-\beta \partial_{x} v(x)+\beta E+\xi(t),
$$

where $\beta$ is the inverse temperature, $\Gamma$ is the inverse relaxation time, $v(x)$ is the random potential, $E$ is the applied uniform field and $\xi(t)$ is the Langevin thermal noise that models the thermal environment,

$$
\left\langle\xi\left(t_{1}\right) \xi\left(t_{2}\right)\right\rangle_{T}=2 \Gamma^{-1} \delta\left(t_{1}-t_{2}\right) .
$$

To distinguish between the average over the thermal noise and disorder averaging we denote the former by the subscript $T$. The inverse relaxation time $\Gamma$ will be set henceforth to 1 for convenience. Making use of the standard approach (see for example Ref.41) one can write the Lagrangian corresponding to Eq.(7) in a form:

$$
\mathcal{L}=-\hat{x}^{2}-i \hat{x}\left(\partial_{t} x-\beta E+\beta \partial_{x} v(x)\right)+\frac{\beta}{2} v^{\prime \prime}(x) .
$$

Further we will set $\beta=1$ measuring energies in the units of temperature. The probability for a particle to go from the point $x_{1}$ to $x_{2}$ is given by the functional integral

$$
P\left(x_{1}, x_{2}\right)=\int D[x(t)] D[\hat{x}(t)] e^{\int d t \mathcal{L}},
$$

and the time a particle spends moving from $x_{1}$ to $x_{2}$ (the delay time) is, correspondingly,

$$
\tau\left(x_{1}, x_{2}\right) \sim P^{-1}\left(x_{1}, x_{2}\right) .
$$

If the temperature is lower than a typical barrier in the system, one can use the saddle point approximation to find $P$

$$
P=e^{A}, \quad A=\int d t \mathcal{L}_{\text {s.p. }},
$$

where $\mathcal{L}_{\text {s.p. }}$ is the value of the Lagrangian (90) at the saddle point trajectory. The delay time $\tau\left(x_{1}, x_{2}\right)$ averaged over the disorder is then

$$
\left\langle\tau\left(x_{1}, x_{2}\right)\right\rangle_{d}=\int D[v] e^{A[v, x]},
$$

with the effective action

$$
A[v, x]=-\frac{1}{2} \int d x_{1} d x_{2} v\left(x_{1}\right) f\left(x_{1}, x_{2}\right) v\left(x_{2}\right)-\int d t \mathcal{L}
$$

where $f$ is the inverse correlation function (1): $\int d x f\left(x_{1}, x\right) u\left(x, x_{2}\right)=\delta\left(x_{1}-x_{2}\right)$. Taking the variational derivatives of the action (14) with respect to $\hat{x}, x, v$ we get the saddle point equations

$$
\begin{array}{r}
-2 \hat{x}+\partial_{t} x-E+\partial_{x} v(x)=0, \\
\partial_{t} \hat{x}-\hat{x} v^{\prime \prime}(x)=0, \\
v(x)=-\int d t \hat{x}(t) u^{\prime}(x-x(t)),
\end{array}
$$

where the variable $\hat{x}$ was redefined $i \hat{x} \rightarrow \hat{x}$. When deriving Eqs.(15,16,17) the last term in Eq(9) was neglected because it gives the $\mathcal{O}(1)$ contribution to the action while the whole instanton action is much larger than one. Following Ref.U, in order to find an instanton solution we have to choose $\hat{x}=\partial_{t} x$. Indeed, this allows to reduce Eqs.(15, 16) to a single equation:

$$
\partial_{t} x=-E+\partial_{x} v(x) .
$$

Assuming that the instanton solution $\hat{x}=\partial_{t} x$ exists in the interval $\left(\eta_{1}, \eta_{2}\right)$ from Eq.(17) we find

$$
v(x)=u\left(x-\eta_{2}\right)-u\left(x-\eta_{1}\right) .
$$

The interval $\left(\eta_{1}, \eta_{2}\right)$, where the instanton solution exists, obviously must lie within the interval $\left(x_{1}, x_{2}\right)$; we will see later, however, that these intervals do not necessarily coincide. Outside the interval $\left(\eta_{1}, \eta_{2}\right)$ one should take the normal solution in the form

$$
\hat{x}=0, \quad \partial_{t} x=E-\partial_{x} v(x) .
$$

The action (14) corresponding to the solution (18) is

$$
A=\frac{1}{2}\left[v\left(\eta_{2}\right)-v\left(\eta_{1}\right)\right]-E\left(\eta_{2}-\eta_{1}\right) .
$$

Since within the interval $\left(\eta_{1}, \eta_{2}\right) \hat{x}=\partial_{t} x$ and outside this interval $\hat{x}=0$, assuming that $\hat{x}$ is continuous, we conclude that on the boundaries of the interval $\left(\eta_{1}, \eta_{2}\right)$ $\hat{x}=\partial_{t} x=0$. Therefore from Eqs.(18, 19) we get the equation

$$
E=-u^{\prime}\left(\eta_{2}-\eta_{1}\right)
$$

which defines the distance $\eta_{2}-\eta_{1}$. Using Eq.(19) the action (21) can be written as

$$
A=u(0)-u(\eta)-E \eta,
$$

where $\eta=\eta_{2}-\eta_{1}$. Note that Eq.(22) corresponds to the extremum of the action (23) with respect to $\eta$, so the final answer for the action can be conveniently presented as the maximal value of the action 


$$
A=\frac{1}{2} K(\eta)-E \eta
$$

where $K(x)$ is given by (2).

The formula (24) allows to find the average delay time for an arbitrary disorder correlation function $u(x)$ and it's concrete realization follows from the specific form of the correlation function $u(x)$.

(i) Short range potential. Let us begin with the simplest case when $u(x)$ monotonically decreases to zero as a function of $|x|$. The corresponding form of the effective potential $v(x)$ is shown on Fig. 1. From Eq.(22) it follows that $\eta \rightarrow \infty$ when $E \rightarrow 0$, thus if $E$ is low enough, the action becomes

$$
A=u(0)
$$

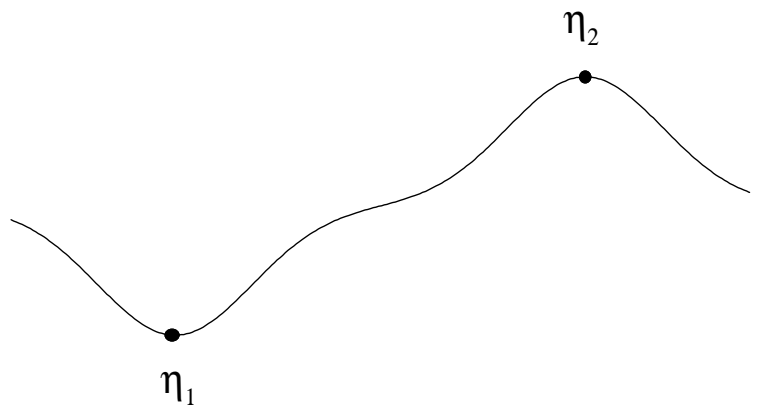

FIG. 1. The instanton solution for the potential $v(x)$ in the case of short range potential.

(ii) Potential with long-range correlations. Let us consider the potential of the form $K(x)=\kappa x^{\gamma}$, with $\kappa>0$ and $0<\gamma<1$. In this case the expression for the action (24) still holds; however, minimizing (24) with respect to $\eta$ we arrive at the action diverging at low fields:

$$
A=(1-\gamma)\left(\frac{\kappa}{2}\right)^{\frac{1}{1-\gamma}}\left(\frac{\gamma}{E}\right)^{\frac{\gamma}{1-\gamma}}
$$

in afull agreement with the exact solution obtained in Ref.e

(iii) 'Extremely correlated' disorder, $\gamma=1$ : This is the well-known Sinai model $K(x)=\kappa x$. Now Eq.(22) is either never satisfied or it is satisfied identically for a special value of the applied field $E=E_{0}=\kappa / 2$. If $E<$ $E_{0}$, the instanton solution exists, but the initial and final points of the instanton motion $\eta_{1}$ and $\eta_{2}$ coincide with points $x_{1}$ and $x_{2}$ respectively, and the action becomes

$$
A=(\kappa / 2-E)\left(x_{2}-x_{1}\right) .
$$

It means that the average time always essentially (exponentially) depends on the boundary conditions. In the case $E>E_{0}$ the instanton solution does not exist.
Distribution function of delay time. The method described above can be simply generalized for a calculation of higher moments of $\tau\left(x_{1}, x_{2}\right)$

$$
\tau_{n}\left(x_{1}, x_{2}\right)=\left\langle\tau^{n}\left(x_{1}, x_{2}\right)\right\rangle_{d} .
$$

The action $A_{n}$ that determines the $n^{\text {th }}$ moment

$$
\left\langle\tau^{n}\left(x_{1}, x_{2}\right)\right\rangle_{d}=e^{A_{n}[v, x]}
$$

is given by

$$
A_{n}[v, x]=-\frac{1}{2} \int d x_{1} d x_{2} v\left(x_{1}\right) f\left(x_{1}, x_{2}\right) v\left(x_{2}\right)-n \int d t \mathcal{L} .
$$

From this equation one can see that $A_{n}[u(x), x]=$ $n A[n u(x), x]$, therefore using Eq.(24) we get the expression for the action $A_{n}$

$$
A_{n}=n^{2} K(\eta) / 2-n E \eta,
$$

that must be maximized with respect to $\eta$. This gives

$$
n K^{\prime}(\eta) / 2-E=0 .
$$

Knowing all moments of $\tau$ one can find the distribution function $P(\tau)$. Indeed, in terms of the distribution function $P(\tau)$, the moments $\tau_{n}$ are defined as

$$
\tau_{n}=\int P(\tau) \tau^{n} d \tau=\int \tilde{P}(Y) e^{n Y} d Y .
$$

Since we use the instanton method to find $\tau_{n}$, with the same accuracy we can use the saddle point approximation in calculation of the integral over $Y$ in Eq.(33). Comparing Eqs.(31,33) we get

$$
\{\ln \tilde{P}(Y)+n Y\}_{Y}=\left\{n^{2} K(\eta) / 2-E n \eta\right\}_{\eta},
$$

where \{\}$_{Y}$ and \{\}$_{\eta}$ mean taking extrema with respect to $Y$ and $\eta$ respectively. Differentiating (34) with respect to $n$ we get

$$
n=\frac{Y+E \eta}{K(\eta)} .
$$

Using Eq.(34) we find the distribution function

$$
\ln \tilde{P}(Y)=-\frac{1}{2} \frac{(Y+E \eta)^{2}}{K(\eta)},
$$

where $\eta$ is defined by the equation

$$
K^{\prime}(\eta)(Y+E \eta)-2 E K(\eta)=0,
$$

following from Eqs.(32,35). Interestingly, this equation also follows form the extremum of the logarithm of the distribution function (36) with respect to $\eta$. 
The distribution function (36) is our main general result. Now we shall analyze it's behavior for different cases introduced above:

(i) In the case of the short-range correlated potential, assuming that the applied field is not too strong, from Eq.(36) we obtain the log-normal distribution

$$
\ln \tilde{P}(Y)=-\frac{Y^{2}}{4 u(0)} .
$$

(ii) In case of the potential $K(x)=\kappa x^{\gamma}, 0<\gamma<1$ from Eq. 37 ) we get

$$
\eta=\frac{\gamma Y}{E(2-\gamma)}
$$

and the distribution function (36) becomes

$$
\ln \tilde{P}(Y)=-\frac{2}{\kappa}\left[\frac{Y}{2-\gamma}\right]^{2-\gamma}\left[\frac{E}{\gamma}\right]^{\gamma} .
$$

(iii) Although in the Sinai case $(\gamma=1)$ the moments of $\tau$ are not defined in the limit of a large system, the Eq.(40) has no singularities when $\gamma=1$. Therefore the distribution function in the Sinai case is given by

$$
P(t)=t^{-2 E / \kappa-1},
$$

which agrees with the earlier result 3 , 1

In conclusion, we have found the asymptotic behavior of the delay time distribution function for a general problem of the Langevin motion of a particle in a onedimensional random potential. The application of our procedure to the Sinai model recovers the earlier results verifying our approach. The developed instanton method allows to derive the distribution function for an arbitrary $1 \mathrm{D}$ random potential, and can serve as an initial step towards a quantitative study of the glassy dynamics of the general multidimensional systems. The method proposed can also be generalized for calculation of other correlation functions which are determined by the contribution from the largest barrier in the system.

Acknowledgments - AVL would like to thank Lev Ioffe for very useful discussions. This work was supported by the U.S. Department of Energy, Office of Science under contract No. W-31-109-ENG-38.

${ }^{1}$ See J.-P. Bouchaud and Antoine Georges, Phys. Rep. 195, 127 (1990)for an extensive review

${ }^{2}$ V. M. Vinokur, J. Phys. (Paris), 47, 1425 (1986)

${ }^{3}$ M.V. Feigel'man and V.M. Vinokur, J. Phys. France 49, 1731 (1989)

${ }^{4}$ D. S. Fisher, P. Le Doussal, and C. Monthus, Phys. Rev. Lett. 80, 3539 (1998)
${ }^{5}$ P. L. Krapivsky and E. Ben Naim, Phys. Rev. E 56, 3788 (1997)

${ }^{6}$ I. Aranson, L. Tsimring, and V. Vinokur, Phys. Rev. Lett. 79, 3298 (1997)

${ }^{7}$ A.V. Lopatin and L.B. Ioffe, Phys. Rev. B 60, 6412 (1999) L. B. Ioffe and D. Sherrington, Phys. Rev. B 57, 7666 (1998)

${ }^{8}$ S. Scheidl, Z. Phyzik B 97, 345 (1995)

${ }^{9}$ P. Le Doussal and V.M. Vinokur, Physica C 254, 63 (1995)

${ }^{10}$ Y. G. Sinai, Theor. Prob. Its Appl., 27, 247 (1982)

${ }^{11}$ K.H. Fischer and J.A. Hertz, Spin Glasses (CambridgeUniversity Press,1993) 